\title{
Prophylactic cranial irradiation or no prophylactic cranial irradiation in metastatic small cell lung cancer: is it a relevant question once again?
}

\author{
Cecile Le Pechoux ${ }^{1}$, Angela Botticella ${ }^{1}$, Antonin Levy ${ }^{1}$, Anne Auperin ${ }^{2}$ \\ ${ }^{1}$ Department of Radiation Oncology, ${ }^{2}$ Department of Biostatistics and Epidemiology, Gustave Roussy Cancer Campus, Villejuif, France \\ Correspondence to: Cecile Le Pechoux. Department of Radiation Oncology, Gustave Roussy Cancer Campus, 114 rue Edouard Vaillant, 94800 \\ Villejuif, France. Email: Cecile.LEPECHOUX@gustaveroussy.fr. \\ Provenance: This is an Invited Editorial commissioned by Section Editor Dr. Di Lu (Department of Thoracic Oncology, Nanfang Hospital, Southern \\ Medical University, Guangzhou, China). \\ Comment on: Takahashi T, Yamanaka T, Seto T, et al. Prophylactic cranial irradiation versus observation in patients with extensive-disease small-cell \\ lung cancer: a multicentre, randomised, open-label, phase 3 trial. Lancet Oncol 2017;18:663-71.
}

Submitted Sep 08, 2017. Accepted for publication Sep 18, 2017.

doi: $10.21037 /$ jtd.2017.10.10

View this article at: http://dx.doi.org/10.21037/jtd.2017.10.10

Even if chemotherapy is the cornerstone treatment of small cell lung cancer (SCLC), this disease has a particular propensity to recur locally and disseminate in the brain. Hence, there have been several randomized trials assessing the role of thoracic and brain radiotherapy (1). Two individual patient data-based meta-analyses showed that thoracic radiotherapy and prophylactic cranial irradiation (PCI) should be part of the therapeutic strategy $(2,3)$. It should be highlighted however, that PCI was a controversial issue for several years before the publication of the PCI overview in 1999, as none of the individual randomised trials could show a significant effect of PCI on overall survival (OS) $(2,4)$. It was also frequently argued that lateoccurring deleterious neurologic effects could outweigh its benefits $(2,4)$. The PCI Overview Collaborative Group meta-analysis represented a turning point in SCLC treatment. Individual patient data of 987 patients $(85 \%$ with limited and $15 \%$ with extensive disease) randomly allocated from 1977 to 1995 to receive or not PCI, were collected and updated. This meta-analysis confirmed that PCI significantly decreased the emergence of detectable brain metastases (from $58.6 \%$ to $33.3 \%$ at 3 years), but most importantly demonstrated a $5.4 \%$ absolute increase in the 3 -year OS rate (from $15.3 \%$ to $20.7 \%$; HR $=0.84$ ). Progress in SCLC treatment in the last 20 years, has thereby consisted essentially in optimisation of chemotherapy and radiotherapy integration both in non- metastatic and metastatic patients. Takahashi and colleagues (5) have to be complimented for reassessing the efficacy of PCI in the treatment of extensive-disease SCLC, and their conclusions challenge what has been the standard of care in metastatic patients since the publication of both the referred meta-analysis and the European Organisation for Research and Treatment of Cancer (EORTC) trial in $2007(2,6)$. According to the EORTC study, addressing the issue of PCI exclusively in 286 metastatic patients, PCI reduced the incidence of symptomatic brain failure and resulted in longer OS in patients who had total or partial response to initial chemotherapy (6). The cumulative risk of symptomatic brain metastases at 1 year was reduced from $40.4 \%$ in the control group to $14.6 \%$ in the PCI group $(\mathrm{P}<0.001)$, and $\mathrm{OS}$ improved (1-year survival: $27.1 \%$ in the PCI group vs. $13.3 \%$ in the control group; $\mathrm{HR}=0.68$; $\mathrm{P}=0.003)$. Therefore, most guidelines recommended PCI should be administered in SCLC patients with good response to initial chemotherapy or chemo-radiotherapy as it reduced the incidence of brain metastases and improved survival (2,7-11).

However, since the publication of the Japanese trial (5), the administration of $\mathrm{PCI}$ in patients with metastatic disease could become again a subject of controversy. In this trial, the cumulative risk of developing brain metastases at 1 year was $32.9 \%$ in the PCI group compared with $59.0 \%$ in the control group $(\mathrm{P}<0.001)$, very similar to the rate observed 
in the meta-analysis. The brain metastases incidence at 1 year of patients included in the PCI overview was respectively $45.5 \%$ among patients who had no PCI $(61.1 \%$ in $\mathrm{ED}$ and $42.6 \%$ in $\mathrm{LD}$ ) and $23.2 \%$ among patients who had PCI (34.1\% in ED and $22 \%$ in LD) (2, Personal communication of 2. Aupérin A). As in the meta-analysis and the EORTC study, PCI reduced two- to three-fold the risks of developing detectable brain metastases, whether symptomatic or asymptomatic, but without a benefit in terms of survival in the Japanese study $(2,5,6)$. Median survival was 10.1 months in the PCI group compared with 15.1 months in the control group $(\mathrm{P}=0.091)$ in the first analysis based on 163 patients, that brought to early termination of the trial. In the final analysis, with a median follow-up of almost 12 months, the gap in terms of median survival was reduced from 5 to 2 months between the two groups: 11.6 months (95\% CI: 9.5-13.3) in the PCI group and 13.7 months (95\% CI: $10.2-16.4$ ) in the observation group (HR =1.27; 95\% CI: 0.96-1.68; $\mathrm{P}=0.094)$. The study thus shows no significant disadvantage of PCI, but there is no impact of PCI on survival. In the Japanese trial, despite the great decrease of BM in the PCI arm, the progressionfree survival was similar in both arms. Because almost all deaths were related to lung cancer, this observation suggests that the other types of progression (i.e., extra-cranial progressions) could have been more frequent in the PCI arm than in the control arm, except if the effect of PCI on brain metastasis concerned only brain metastases that would occur concomitantly or after extra-cranial progression in this trial population, which is unlikely. In the EORTC trial, the disease-free survival was significantly improved in the PCI arm as compared to the control arm. If extracranial progressions were really more frequent in the PCI arm than in the control arm in the Japanese trial, it could explain the absence of OS improvement, and the most likely reason for an increase of extra-cranial progressions in the PCI arm as compared to the control arm would be "by chance". It would have been of high interest to have the patterns of failure (brain progression versus extracranial progression) in both arms (as first events, each alone and both concomitantly) of both studies to interpret at best the results of both trials. One would expect that treating undetectable brain metastases would have more impact on survival than treating gross disease yielding symptoms. In the EORTC study, the authors explained the survival improvement by the fact that more patients in the PCI group could have second line treatment (6). In the Japanese study, it should be highlighted that the proportion of patients with second line treatment (around 88\%) was similar in both arms, thereby not affected by delivery of PCI (5). These results are then very different from previous studies where less than $50 \%$ patients treated for extensive disease, were candidates for second-line chemotherapy with poor response rates (10\% to $25 \%)(6,8)$.

But is this absence of improved survival a major drawback? We should not forget PCI is the only treatment that significantly reduces the development of brain metastases whether symptomatic as proven by the EORTC trial and/or asymptomatic as shown in the Japanese trial and all PCI trials $(5,6)$. Compared to extra-cerebral distant failure, the occurrence of brain metastases has a considerable impact on the quality of life (QoL) of lung cancer patients and represents a significant economic burden (12). It is interesting to highlight the results of one of the largest trials included in the meta-analysis, where all patients had brain CT scan before randomisation and about $20 \%$ of patients included had metastatic disease (13). With a follow-up brain CT scan every 6 months, the total brain metastases rate at one year was estimated at around $20 \%$ in the PCI group and $55 \%$ in the control group, and respectively $40 \%$ and $67 \%$ at 2 years. Even if there was no effect of PCI on survival (2-year survival of $29 \%$ in the PCI group and $21.5 \%$ in the control group, $\mathrm{P}=0.12$ ), the authors concluded that the cost/benefit balance was in favour of PCI: a threefold decrease in the rate of brain metastases, low cost and mild toxicity, as well as a possible potential effect on OS (14). In the Japanese trial, with brain MRIs performed every 6 months, the brain failure rates at 18 months are comparable, decreased from $63.8 \%$ to $40.1 \%$ (5). One can assume that MRIs allow discovering brain metastases six months earlier. Thereby, there does not seem to be any different propensity for brain failure among Japanese and European patients, explaining differences of outcome. The much higher frequency of brain metastases observed in the Japanese study is mainly attributable to the detection of asymptomatic brain metastases by MRI.

We dispose now of two randomised trials evaluating PCI specifically in SCLC patients with metastatic disease, with conflicting results $(5,6)$. How can we reconcile data from these two studies? The inclusion criteria are quite different in both trials, the primary end-point being the rate of symptomatic brain metastases in the EORTC trial (6) and OS in the Japanese trial (5). In the EORTC study, cranial irradiation for symptomatic BM was administered in 2 of 24 patients in the PCI group (8.3\%), as compared with 35 of 59 patients in the control group (59.3\%). In the 
Japanese study, cranial irradiation (mostly stereotactic) for BM was performed for $46 \%$ (25/44 patients) of patients in the PCI group and in $83 \%$ patients (64/77 patients) in the control group. This shows once again the burden of brain failure (69\% patients in the control group develop BM). Median interval from study enrolment to radiotherapy for BM was 384 days in the PCI group, double time as that of the observation group (193 days). Takahashi et al. consider the cost/benefit balance is not in favour of PCI because of the absence of impact on survival and despite a lower rate of brain metastases and low toxicity in terms of adverse events at 3 months and neurotoxicity evaluated with mini mental state examination (no difference in terms of scores at 12 and 24 months). Undergoing brain MRI every 3 months can be quite stressful and have an impact on QoL of these patients who have a limited life expectancy. Treatment cost and impact on QoL have not been addressed in this trial. In the EORTC study, patients underwent a QoL evaluation: there was a negative impact of PCI on functioning scales but this was moderate (15). Patients in the PCI arm had significantly more prolonged hair loss and increased fatigue at 6 weeks and 3 months. Longer term data were limited, as median survival was poor. Other studies have been able to study longer term results $(16,17)$ in terms of neurocognitive deficit or QoL showing a decline most particularly of memory, but considered mild by most patients $(16,18)$. These studies as well as others have also shown that this decline was more frequent and severe in elderly patients; such analysis was not performed in the Japanese trial where more than $47 \%$ of patients were 70 or older. In a phase III trial evaluating PCI in non-metastatic NSCLC, early changes at 3 months after PCI were more important and significant than later changes; a subacute recovery could be observed in several tests evaluating neurocognitive functions including MMSE used in the Japanese trial (17). These observations of early decline especially in elderly patients followed by neurocognitive recovery after 3 months are of importance when a clinician is confronted with delivery of PCI in metastatic patients whose median survival does not exceed 12 months. However it will be important to implement results of several ongoing studies exploring ways to decrease neurotoxicity such as hippocampus sparing PCI $(19,20)$.

Another issue that deserves some discussion is that patients seem to have better outcome in the Japanese trial: the one-year survival rate is respectively $13.3 \%$ in the EORTC study and $53.6 \%$ in the Japanese study among patients who had no PCI and the one-year survival rate is respectively $27.1 \%$ in the EORTC study and $48.4 \%$ in the Japanese study among patients who had PCI. Observed differences in efficacy outcomes between these two trials testing similar treatment regimens may be due to several factors, including differences in eligibility criteria, patient selection, demographics, and treatment regimens. Another reason for this divergence of outcomes may be related to host-related genetic differences such as molecular differences in SCLC between Asian and nonAsian populations, as suggested by studies in metastatic disease $(21,22)$. Some of the possible explanations for this different outcome have been highlighted by Takahashi et al. and have led them to initiate this trial in Japan. As opposed to the EORTC trial, brain imaging before enrolment was required, as well as cisplatin containing regimens known to be associated with higher response rates and probability of survival than regimens without cisplatin (23) and detailed assessment of response. Considering the strict selection of patients in the Japanese trial, based on imaging within 4 weeks from randomisation, we can consider it is really a PCI as brain imaging and assessment of thoraco-abdominal response were performed in all patients. The percentage of patients who could have a fourth line treatment (26-36\%) is a compelling evidence that this group of patients whose median age was 69, was highly selected and may not be representative of all SCLC patients with extensive disease eligible for PCI. In the EORTC study, patients had no baseline brain evaluation unless they were symptomatic, and assessment after chemotherapy was based on chest evaluation. We can hypothesize that both patients with detectable asymptomatic brain metastases as well as patients without detectable metastases, were included in this trial, which should be considered more a trial of consolidation whole brain irradiation rather than a strict PCI trial. In summary, Takahashi et al. conclude that PCI should be omitted on the condition that patients with extensivedisease small-cell lung cancer are periodically assessed with brain MRI, every 3 months. However, they highlight the fact that in countries other than Japan, "physicians should be cautious when extrapolating the results of this study to general practice". Taking into consideration the results of these two trials, and waiting the results of eventual new trials, we should not forget that brain failure in SCLC is often multiple and can hardly be treated with surgery or SRT as opposed to NSCLC. It may be salvaged by whole brain irradiation without compromising OS. However the impact on QoL of such brain failure, in a population with poor life expectancy should also be taken into consideration. In 
determining the net clinical benefit of PCI in patients with extensive disease, both the quantity and quality QoL should be considered and discussed with patients. A new trial would be warranted to evaluated optimised PCI in good responders after CT or CTRT undergoing MRI staging, especially at an era of possible new systemic treatment strategies such as immunotherapy.

\section{Acknowledgements}

None.

\section{Footnote}

Conflicts of Interest: The authors have no conflicts of interest to declare.

\section{References}

1. Woolf DK, Slotman BJ, Faivre-Finn C. The Current Role of Radiotherapy in the Treatment of Small Cell Lung Cancer. Clin Oncol (R Coll Radiol) 2016;28:712-9.

2. Aupérin A, Arriagada R, Pignon JP, et al.Prophylactic cranial irradiation for patients with small-cell lung cancer in complete remission. Prophylactic Cranial Irradiation Overview Collaborative Group. N Engl J Med 1999;341:476-84.

3. Pignon JP, Arriagada R, Ihde DC, et al. A meta-analysis of thoracic radiotherapy for small-cell lung cancer. $\mathrm{N}$ Engl J Med 1992;327:1618-24.

4. Péchoux CL, Sun A, Slotman BJ, et al. Prophylactic cranial irradiation for patients with lung cancer. Lancet Oncol 2016;17:e277-93.

5. Takahashi T, Yamanaka T, Seto T, et al. Prophylactic cranial irradiation versus observation in patients with extensive-disease small-cell lung cancer: a multicentre, randomised, open-label, phase 3 trial. Lancet Oncol 2017;18:663-71.

6. Slotman B, Faivre-Finn C, Kramer G, et al. Prophylactic cranial irradiation in extensive small-cell lung cancer. N Engl J Med 2007;357:664-72.

7. Meert AP, Paesmans M, Berghmans T, et al. Prophylactic cranial irradiation in small cell lung cancer: a systematic review of the literature with meta-analysis. BMC Cancer 2001;1:5.

8. Früh M, De Ruysscher D, Popat S, et al. Small-cell lung cancer (SCLC): ESMO Clinical Practice Guidelines for diagnosis, treatment and follow-up. Ann Oncol 2013;24
Suppl 6:vi99-105.

9. Jett JR, Schild SE, Kesler KA, et al. Treatment of small cell lung cancer: Diagnosis and management of lung cancer, 3rd ed: American College of Chest Physicians evidence-based clinical practice guidelines. Chest 2013;143:e400S-19S.

10. Lung cancer: diagnosis and management. Available online: https://www.nice.org.uk/guidance/CG121/chapter/1Guidance (accessed August 13, 2017).

11. Ettinger DS, Wood DE, Aisner DL, et al. Non-Small Cell Lung Cancer, Version 5.2017, NCCN Clinical Practice Guidelines in Oncology. J Natl Compr Canc Netw 2017;15:504-35.

12. Peters S, Bexelius C, Munk V, et al. The impact of brain metastasis on quality of life, resource utilization and survival in patients with non-small-cell lung cancer. Cancer Treat Rev 2016;45:139-62.

13. Arriagada R, Le Chevalier T, Borie F, et al. Prophylactic cranial irradiation for patients with small-cell lung cancer in complete remission. J Natl Cancer Inst 1995;87:183-90.

14. Arriagada R. Re: Prophylactic cranial irradiation for patients with small-cell lung cancer. J Natl Cancer Inst 1995;87:766; author reply 767 .

15. Slotman BJ, Mauer ME, Bottomley A, et al. Prophylactic cranial irradiation in extensive disease small-cell lung cancer: short-term health-related quality of life and patient reported symptoms: results of an international Phase III randomized controlled trial by the EORTC Radiation Oncology and Lung Cancer Groups. J Clin Oncol 2009;27:78-84.

16. Gondi V, Paulus R, Bruner DW, et al. Decline in tested and self-reported cognitive functioning after prophylactic cranial irradiation for lung cancer: pooled secondary analysis of Radiation Therapy Oncology Group randomized trials 0212 and 0214. Int J Radiat Oncol Biol Phys 2013;86:656-64.

17. Sun A, Bae K, Gore EM, et al. Phase III trial of prophylactic cranial irradiation compared with observation in patients with locally advanced non-small-cell lung cancer: neurocognitive and quality-of-life analysis. J Clin Oncol 2011;29:279-86.

18. Le Péchoux C, Laplanche A, Faivre-Finn C, et al. Clinical neurological outcome and quality of life among patients with limited small-cell cancer treated with two different doses of prophylactic cranial irradiation in the intergroup phase III trial (PCI99-01, EORTC 22003-08004, RTOG 0212 and IFCT 99-01). Ann Oncol 2011;22:1154-63.

19. Redmond KJ, Hales RK, Anderson-Keightly H, et al. Prospective Study of Hippocampal-Sparing Prophylactic 
Cranial Irradiation in Limited-Stage Small Cell Lung Cancer. Int J Radiat Oncol Biol Phys 2017;98:603-11.

20. Belderbos J, Schagen S, Walraven I, et al. In Regard to Redmond et al. Int J Radiat Oncol Biol Phys 2017;99:238-9.

21. Noda K, Nishiwaki Y, Kawahara M, et al. Irinotecan plus cisplatin compared with etoposide plus cisplatin for extensive small-cell lung cancer. N Engl J Med 2002;346:85-91.

22. Hanna N, Bunn PA Jr, Langer C, et al. Randomized phase

Cite this article as: Le Pechoux C, Botticella A, Levy A, Auperin A. Prophylactic cranial irradiation or no prophylactic cranial irradiation in metastatic small cell lung cancer: is it a relevant question once again? J Thorac Dis 2017;9(11):41574161. doi: $10.21037 / j t d .2017 .10 .10$
III trial comparing irinotecan/cisplatin with etoposide/ cisplatin in patients with previously untreated extensivestage disease small-cell lung cancer. J Clin Oncol 2006;24:2038-43.

23. Pujol JL, Carestia L, Daurès JP. Is there a case for cisplatin in the treatment of small-cell lung cancer? A meta-analysis of randomized trials of a cisplatin-containing regimen versus a regimen without this alkylating agent. Br J Cancer 2000;83:8-15. 